\title{
Risk perception in emergency planning environments
}

\author{
A. Pratelli \\ Department of Civil and Industrial Engineering, University of Pisa, Italy
}

\begin{abstract}
Catastrophic events which are able to generate great emergency situations involving very large areas and huge networks are usually characterized by very low values of occurrence probability. This leads us to believe that individual risk perception is consequently and most likely affected by errors and it is also rather far from the effective risk level. Therefore, a priori assignment of an event risk factor is not very correct, because it can lead to large misevaluations. The risk measure of an event is a research field where some concepts belonging to psychometrics, physics and sociology are often interacting, if not mutually overlapped.

This paper deals with a relatively new approach to individual risk perception and evaluation based on Prospect Theory and shows its applications for disaster and emergency management in a transport network. The main differences and advantages with other more traditional approaches are also displayed and discussed. Finally, some practical examples are referred to in order to support the strengths of Prospect Theory potential applications.

Keywords: risk perception, Prospect Theory, emergency management, decision making.
\end{abstract}

\section{Introduction}

Any individual person and every social group have a good knowledge of their regular exposure to different risks. This fact is normally accepted as part of life even if, when faced with a certain measure of risk exposure, everyone expects to receive a counterpart in terms of a corresponding rate of granted or expected benefits. There are several research works dealing with the "corresponding rate" between risk and benefit, each one suggesting a different trade-off method [1-4]. 
Individuals react to a perceived risk and, of course, the reaction strength is linked to such a type of risk in some extent. Nevertheless the individual perception strength can be over or under estimate, until to generate scepticism and adverse behaviour in respect to any mitigation and prevention countermeasure.

The risk measure of an event is a research field where some concepts belonging to psychometrics, physics and sociology are interacting and mutually overlapped.

Many events are well-known through their data time series, e.g. road accidents. Some cases require complex statistics analysis in order to go back to find the probability of the event, e.g. risk of smoking and alcohol abuse.

Other types of events have no useful statistics data, because they are extremely rare or absolutely new. These are cases related to nuclear accidents, or to genetic code manipulation, and all the instances without practical experience where risk determination is only relayed to theoretical models.

From the individual's point of view, it is fairly common to speak about "acceptable risk". This to signifies that individuals are disposed to suffer certain risk exposure in view of a given revenue or satisfaction. Such an attitude is subject to a suddenly change when the individuals feel to have lost any control power on the event and their behaviour shifts from "risk seeking" to "risk adverse".

Ruinous events which are able in determining some emergency situations in large areas are generally characterized by fairly low values of probability of happening. This way, one is often likely led to believe that individuals risk perception is biased and quite far from the effective risk level. It is not too much correct any a priori risk value assignment, but it is better to test its importance through well-suited surveys and methods.

A good methodology for the above goals is the so-called Prospect Theory, or PT, firstly proposed by Kahneman and Tversky [5] in business and economics applications. The main characteristic of this descriptive technique is to explain how individuals choose among alternatives when outcomes associates with those alternatives are probabilistic or deeply uncertain in nature. Then choices that individuals make under situations of risk and uncertainty are influenced by some psychological factors and environmental suggestions, sometimes called "mind tunnels" [6].

Prospect Theory has been applied with great success to applications in several fields as business, economics, medicine, law, finance, political science and transport. In this last sector PT has been applied to estimate how customers evaluate unreliability in public transport, risk of accident and congestion, uncertainty in parking search or risk of missing a train connection [7].

All those results and applications have highlighted how individuals have a biased perception as many very low as very high probability of events, while probability closer to $50 \%$ are perceived quite properly [8].

In the following is presented the opportunity of using Prospect Theory, as an alternative to traditional Expected Utility Theory, for modelling decisions under 
conditions of risk and emergency instances, by emphasizing its capacity of better reproduce the role of psychological factors.

\section{Traditional approaches based on Expected Utility Theory}

Expected Utility Theory, or EU, has been the preeminent model for rational decision making since the second half of twentieth century, and the concept that individuals attempt to maximize their own preferences is largely intuitive.

Following de Palma et al. [9], let $X=(E 1: x 1, \ldots, E n: x n)$ denote a prospect. The $E j, j=1, \ldots, n$ denote possible events of which exactly one is true and the others are not true, and it is unknown to us which one is true. The quantity $x j$ designates an amount of money (or any similar source of utility), which is the outcome of the prospect if $E j$ is true, $j=1, \ldots, n$. When choosing from multiple prospects, the decision maker selects one that equally or more than dominates all others. Because we do not know for sure which event is true, we do not know for sure what outcome will result from a prospect. This is reflected by the term decision under uncertainty.

Decision makers maximize their expected utility:

$$
E U=\sum_{j=1}^{n} P(E j) U(x j)
$$

and $X$ will be chosen when compared to $Y$ if and only if:

$$
\sum_{j=1}^{n} P(E j)[U(x j)-U(y j)] \geq 0
$$

where: $U($.$) is the utility function and the P(E j)$ 's are (subjective) probabilities.

A crucial property of this formulation is that probabilities are processed in a linear manner. They need not be objective probabilities but may instead reflect subjective judgments of the decision maker. If objective probabilities are common knowledge, then the subjective probabilities agree with them (under mild assumptions), and we often suppress the events $E j$, writing $p j$ for $P(E j)$.

However, the conditions required for classic Expected Utility Theory, seldom, if never, exist in real world environmental hazards and disaster planning problems: full knowledge of the hazards and disaster planning alternatives, project costs, and objective utility. These assumptions are simply unrealistic and EU Theory has been widely criticized, and the broad consensus is that the descriptive value of EU Theory is limited. A central issue in this popular decision making paradigm involves the individual's risk profile, in the sense of risk seeking or risk adverse [10]. An individual strictly preferring a sure thing to a gamble with the same expected value is called risk adverse. If an individual has decreasing marginal utility from wealth and maximizes his/her expected utility, he/she must be risk adverse.

Moreover, there are many important situations observed in the decision making of individuals that not explained by the Expected Utility Theory. People are able to process relatively little of the information available to them. This fact 
involves a certain "bounded rationality" in that decisions occurs in limited time frames and individuals are not aware of (or unable to acquire) all the information required to make a decision. The term "bounded rationality" implies somewhat less than "perfect" rationality.

Optimization may be misunderstood in emergency and disaster planning situations often characterized by high uncertainty, non-linear relationships, dynamics in preferences and changes of decision makers over the time horizon.

Bounded rationality approach recognizes that decision makers are not completely rational in term of traditional EU Theory. Moreover, in the realworld of emergency and disaster planning, both companies and individual decision makers rarely examines all alternatives or pay equal attention to all potential relevant variables, but essentially proceed using heuristics behaviour to cope with complexity.

\section{The Prospect Theory based approach}

Prospect Theory is a behavioural economic theory that describes the way individuals chose between probabilistic alternatives that involve risk, where the probabilities of outcome are known. The theory states that individuals make decisions based on the potential value of losses and gains rather than the final outcome, and that individuals evaluate these losses and gains using certain heuristics. The model tries to represent real-life choices, rather than optimal decisions.

The Prospect Theory, or PT, was developed by Kahneman and Tversky [5] as a psychologically more accurate description of the decision making process. They challenged the explicit rules of rational decision making theory by noting that choices that individuals make under situations of risk and uncertainty show several characteristics that are inconsistent with the classic expected utility principles.

Prospect Theory is herewith firstly described in a straightforward manner by an illustrating example [11].

Accidentally, the theory was first tested in an experiment that was an emergency management decision-making problem [5] as follows:

- Assume that in our emergency preparedness planning we are faced with a disease affecting 600 people and we have two choices:

1) Program A, by which 200 of the 600 people will be saved;

2) Program $B$, in which there is a $1 / 3$ chance that all the 600 will be saved and a $2 / 3$ chance that nobody will be saved.

(The reader can think about these two programs considering which he/she would choose before continuing).

Prospect Theory predicts that many of us, just like those who responded to Kahneman and Tversky's experiment, will choose program A. From a rational point of view, the end results of both choices are the same: 200 will be saved $(600 \times 1 / 3+0 \times 2 / 3=200)$. Therefore, we should actually have a neutral reaction to these two choices. 
Before explaining why this happens let us try another experiment. This time imagine that we are given the following emergency preparedness options and are asked to choose between them:

1) Program C, whereby 400 people will die;

2) Program $D$, in which there is a $1 / 3$ chance that nobody will die, and a $2 / 3$ chance that all 600 people will die.

(Again, the reader can consider the above last two choices making one choice before continuing).

According to PT's predictions, most of us - nearly 80 percent - would choose Program D. Nevertheless, the end results of these choices are again the same: 400 people will die $(0 \times 1 / 3+600 \times 2 / 3=400)$, indicating that we are acting irrationally because we are behaving against rational decision-making principle; i.e., if we are asked to choose among two identical apples, we should not prefer one over another. To understand why this happen, let's go back to the experiments. In the first two emergency management choices, the majority of interviewed respondents choose Program A because human beings certainty over uncertainty, and Program A seems more plausible to them. By choosing this option, individuals do not take any risk and thus surely save 200 people. In the second instance, large part respondents choose Program D because they naturally think to avoid the loss of 400 people: unlike the reaction to the first experiment, this time they prefer taking the risk.

Based on a number of such experiments, Kahneman and Tversky formulated two main propositions. First, human beings are risk adverse toward gains saving lives in choice A - and risk seekers toward losses - loss of lives in choice D.

Second, losses are more threatening to people than gains are appealing: the magnitude of a negative utility (sadness) from a loss exceeds the positive utility (happiness) of a gain of the same size.

These explanations not only solve some of the academic paradoxes existing in the literature about traditional decision-making models based on Expected Utility Theory, they also provide answers to a number of questions that many emergency managers encounter day-by-day in practice.

\subsection{Decision model}

Prospect Theory involves two stages in the decision making process: a first stage of editing, and a second stage of evaluation [12].

\subsubsection{Editing}

The editing phase is the preliminary mental analysis of the offered choices, or prospects, in which individuals try to organize and reformulate the options in order to simplify the decision problem. The editing phase involves several operations that transform the outcomes and probabilities to the offered choices. These operations are applied to each choice separately (Fig. 1) as described in the following: 


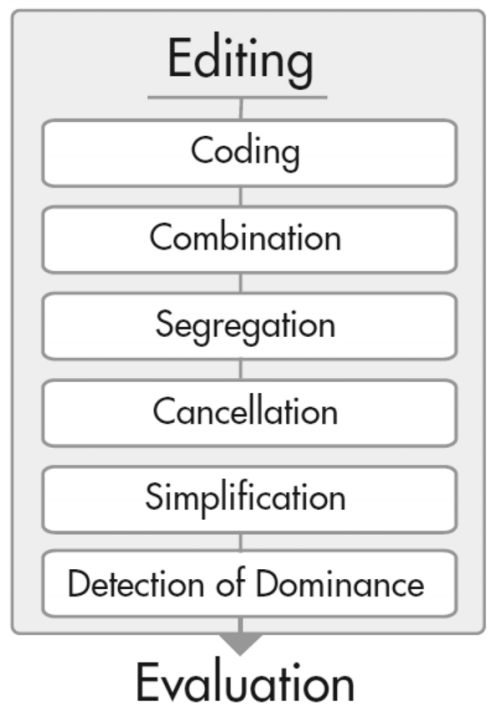

Figure 1: $\quad$ Prospect Theory decision making model steps [11].

a) Coding: individuals try to view outcomes as losses or gains in respect to a reference point corresponding to their current position, instead of final states of wealth.

b) Combination: individuals try to simplify the choices offered by combining options with very similar outcomes. For example, if a person live in a town with $10 \%$ of chance of dying in an earthquake and $10 \%$ of dying in a fire in a given year, that person will evaluate potential moves to a town with $10 \%$ likelihood of dying in a flood, assuming a $20 \%$ chance of dying in an earthquake or fire by staying in the same town [11].

c) Segregation: individuals also segregate choices which contain a riskless component from those that have a risky component. For example, the prospect between of "receiving $€ 20,000$ of damage with a probability of $20 \%$ " and "receiving $€ 60,000$ of damage with a probability of $80 \%$ " can be decomposed into a sure loss of $€ 20,000$ and "losing $€ 40,000$ with a probability of $80 \%$ " [11].

d) Cancellation: if faced to a set of three or more prospects, individuals also try to drop off components that are shared by the offered prospects. For example, if evacuation and shelter in a place both involves 10 fatalities, this number of fatalities will be eliminated from the decision model [11].

e) Simplification: individuals also try to simplify the options and this is the process of rounding probabilities/outcomes of a choice. For example, a $51 \%$ probability of receiving $€ 29,000$ damages is likely to be evaluated as a $50 \%$ chance of receiving $€ 30,000$ damages [11]. Furthermore, simplification is extended to the discarding of extremely unlikely (unattainable or impossible) outcomes. 
f) Detection of Dominance: this step looking for dominated alternatives in the choices offered which are rejected without further evaluation.

\subsubsection{Evaluation}

Once the editing phase is complete the decision maker is expected to evaluate each one of the edited prospects. In the evaluation stage, individuals behave as they would compute a value (utility), based on the potential outcomes and their respective probabilities, and then choose the alternative having the highest value.

According to Kahneman and Tversky [5], "the overall value of an edited prospect denoted $V$, and is expressed in terms of two scales, $w$ and $v$ ".

The first scale associates with each probability $p$ a decision weight, $w(p)$, which reflects the effect of the probability on the total value of the prospect.

The second scale, $v$ assigns to each outcome $x$ a number $v(x)$, which is the subjective value of the outcome. Since outcomes in Prospect Theory are defined relative to a reference point $v$ measures the value of deviations from the reference point (thus $v$ represents gains or losses).

The formula that is assumed for the evaluation stage is given by:

$$
U=\sum_{i=1}^{n} w(p i) v(x i)=w(p 1) v(x 1)+w(p 2) v(x 2)+\ldots \ldots+w(p n) v(x n)
$$

where the function $w(p)$ is a probability weighting function and expresses that individuals tend to overreact to small probability events, but underreact to medium and large probabilities (Fig. 2).

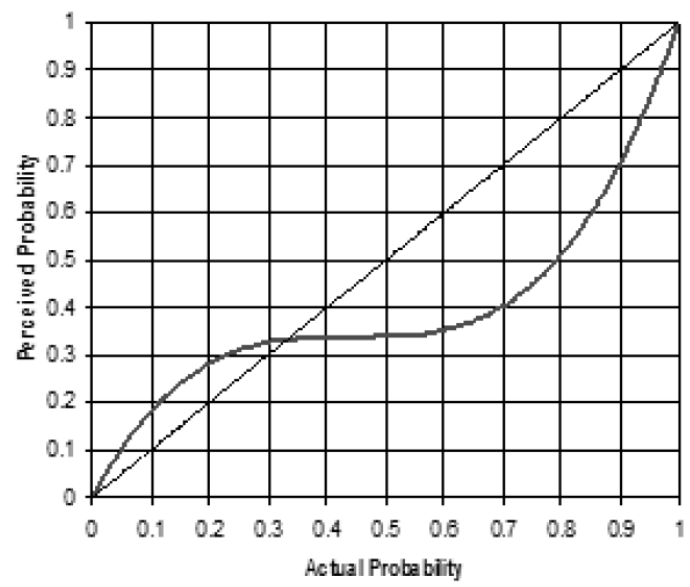

Figure 2: $\quad$ Example of weighted function in Prospect Theory. 


\section{Psychological effects in emergency and disaster planning}

In each of the above decision phases, there are several psychological effects that shape individuals decisions under risk and uncertainty situations and could end up irrational decisions. These effects are listed below, and potential implications of each effect for emergency and disaster planning are discussed in brief.

\subsection{Certainty effect}

The first psychological effect is called certainty effect. People underweight outcomes that are merely probable in comparison with whose are obtained with certainty. This tendency contributes to risk aversion in choices involving sure gains and to risk seeking in choices involving sure losses. In taking decisions where physical risk are involved the certainty effect means that people prefer the total elimination of risk instead reducing it. This effect is an important contributing factor for a considerable number of human errors causing major technological disasters. The certainty effect may to a degree explain why voluntary earthquake mitigation measures are unattractive: their short-term costs may be counted as sure losses and outweigh the long-term, rather uncertain benefits. Moreover, consider a case when planners need to make a choice between two projects: the project that has no environmental risk or eliminate the risk will be preferred to the other project that reduces the risk. Planners should expect people to prefer certain hazards and disaster planning outcomes as compared to uncertain outcomes even if the total probability value for these last seems more [10].

\subsection{Framing effect}

The second psychological effect is called framing effect. Prospect Theory suggests that the way that choices are presented has an impact on our decisions, even though their outcome might be the same. People sometimes make different choices when the same problem is presented to them in different ways. For example, the possible outcome of a gamble can be framed either as gains or losses relative to the status quo or as asset positions that incorporate initial wealth. In emergency and disaster planning, frames can be made differently in a wide range and could have significant implications. Just like any decision problem, it is possible to frame a given scenario in more than one way by using different writing, verbal and visual techniques. Framing is also an important issue in disaster planning negotiations, especially when different stakeholders participate in some forms of negotiation. Some players, under some conditions, can manipulate other actors into a decision or policy choice that is more desirable to them, This can be done by acting on the risk attitude of other actors so that they are inducted to select a different point or opinion. It is easily argued that framing poses some particularly political and ethical dilemma to planners that are faced to several questions. It is a very wide field, because a challenge for emergency and disaster planning theory is to devise tools to accurately recognize frames at work in specific situations (e.g., for more see [14]). 


\subsection{Reference point effect}

The third psychological effect is the so called reference point effect. Depending on the reference point, our decisions might be different. The most recent examples are September 11, 2001, and Hurricane Katrina. Nowadays most people's reference points are these two events, which have changed our decision environment significantly, even though there have not been significant physical changes in our risk conditions [13]. In Prospect Theory the variation of a reference point therefore determines whether a given outcome is considered a loss or a gain. As a result, we might either overestimate or underestimate the risk and thus make irrational decisions. Unfortunately, in most cases people's reference point is the status quo, and people tend to anchor to it. Empirical evidence shows that individuals are reluctant to move away from the status quo even though there may be substantial gains to them by doing so. That is why they do not take risk mitigation and preparedness measures seriously [11].

In emergency and disaster planning reference point could be different things: current state of the community (status quo), future state of the community and previous state of the community, hazard and disaster planning goals, another reference community. Generally reference points are representing expectations based on the past experiences, norm of fairness and social customs and as such people and planners' expectations are shaped by past experiences, comparisons between communities, special rules and guidelines, and the future as well [15].

\subsection{Availability effect}

The final effect is called availability effect. By way of example, many firms and individuals without insurance at the time of a disaster will wish to buy insurance immediately afterward and then cancel their policies several years later if they haven't suffered a loss. This behaviour is principally attributable to the availability bias and saliency of the event. Insurance providers, by contrast, will often withdraw from the market or quote prohibitively high premiums to anyone who applies for new insurance after a disaster, even though the expected value benefits they would pay out in the next period should be affected only modestly by the occurrence of the catastrophic event. Like the victims of disasters, they fixate on the event itself and forget about the loss probability [11]. This effect is also taken into account by many companies that have run to offer new costly house insurance policies all over Italy after the ruinous earthquake shocks that destroyed several buildings in Emilia (central Italy) during Summer 2012. In brief, the availability effect shows how our decisions are impacted by recent events that are easily available in our minds. The decisions of emergency planners are often affected by the availability effect. In coping with cases there recently have been disasters with significant media coverage (e.g., an explosion in a chemical factory), precaution is high for certain types of planning proposals even though the actual physical condition or risk might not have changed significantly. The availability effect can also impact the forecasts of planners and decision makers who will give higher probability of recurrence to more recent 
events. Thus the recent attention of emergency decision makers to risk of terrorism due to the availability impact created after September 11, 2001.

\section{Concluding remarks}

Prospect Theory is a well suited theory of decision making under risk and uncertainty conditions, by emphasizing the role of psychological factors. Emergency, hazards and disaster planning decisions are mainly formulated both on technical criteria, and on the basis of individual judgement and values. Such decisions vary between different players and under environmental situations. Prospect Theory potential is able to consider explicitly the impact of values, emotions and risk perception in the decision making process. Finally, it looks very important to emergency planning and disaster analysis to help a planner be aware of possible biases and ensuring that decisions he makes are rational and discreet.

\section{References}

[1] Winkler R.L. (1968) The consensus of subjective probability distributions. Mgmt. Sci. 15, B-61 through B-75.

[2] Starr C. (1969) Social benefit versus technological risk. Science 165.

[3] Starr C., Rudman R. and Whipple C. (1976) Philosophical basis for risk analysis. Annual Review of Energy, 662-629.

[4] Rowe W.D. (1977) An Anatomy of Risk. John Wiley and Sons, New York.

[5] Kahneman D. and Tversky A. (1979) Prospect theory: an analysis of decision under risk. Econometrica 47, 263-291.

[6] Piattelli Palmarini M. (1994) Inevitable illusions. John Wiley and Sons, New York.

[7] Rietveld P., Bruinsma F.R. and Van Vuren D.J. (2001) Coping with unreliability in transport chains: a case study for The Netherlands. Transportation Research A 35 (6), 539-559.

[8] Wu G. and Gonzalez R. (1996) Curvature of the probability weighting function. Mgmt. Sci. 42, 1676-1690.

[9] de Palma A., Ben Akiva M. et al. (2008) Risk, uncertainty and discrete choice models. Springer Science, Berlin.

[10] Asgary A. and Levy J. (2009) A review of the implications of Prospect Theory for natural hazards and disaster planning. International Journal of Environmental Research, Vol. 3 (3), pp. 379-394.

[11] Asgary A. (2005) What emergency managers need to know about prospect theory. Emergency Management Canada, Vol. 1 (4), pp. 37-39.

[12] Chavez B., Huang Y., Mallavarapu T. and Wang Q. (2012) Notes on: Prospect Theory: an analysis of decision under risk (Kahneman and Tversky, 1979). ECON 206, Duke University.

[13] Sacco K., Galletto V. and Blanzieri E. (2003) How has the 9/11 terrorist attack influenced decision making? Appl. Cognitive Psychol. 17, pp. 11131127. 
[14] Kaufman S. and Smith J.L. (1999) Framing and reframing in land use change conflicts. Journal of Architectural and Hazards and Disaster Planning Research, 16, pp. 164-180.

[15] Shalev J. (2002) Loss aversion and bargaging. Theory and Decision, 52, pp. 201-232. 